\title{
STRUCTURE DIAGRAMS FOR PRIMITIVE BOOLEAN ALGEBRAS
}

\author{
JAMES WILLIAMS ${ }^{1}$
}

\begin{abstract}
If $S$ and $T$ are structure diagrams for primitive Boolean algebras, call a homomorphism $f$ from $S$ onto $T$ right-strong iff whenever $x T f(t)$, there is an $s$ such that $f(s)=x$ and $s S t$; let $R S E$ denote the category of diagrams and onto right-strong homomorphisms. The relation "S structures $B$ " between diagrams and Boolean algebras induces a 1-1 correspondence between the components of $R S E$ and the isomorphism types of primitive Boolean algebras. Up to isomorphism, each component of $R S E$ contains a unique minimal diagram and a unique maximal tree diagram. The minimal diagrams are like those given in a construction by William Hanf. The construction which is given for producing maximal tree diagrams is recursive; as a result, every diagram $S$ structures a Boolean algebra recursive in $S$.
\end{abstract}

1. Right-strong epimorphisms. We shall use the following notation for binary relations: If $P, Q$ are relations, then $P Q=\{\langle x, z\rangle \mid j y: x P y$ and $y Q z\} ; \operatorname{dom} P=\{x \mid \exists y: x P y\} ;|P|=\{x \mid \exists y: x P$ y or $y P x\} ; P^{\smile}=\{\langle y, x\rangle \mid$ $x P y\}$; if $P$ is a function, then $P(x)=y$ iff $x P y$; in any case $P[y]=$ $\{x \mid x \quad P y\}$. While this last condition is perhaps unconventional, it is at least compatible with the notation for Boolean algebras: $\mathfrak{A}[a]=\{b \in|\mathfrak{A}| \mid b \leq$ a).

A Boolean algebra $B$ is pseudo-indecomposable iff whenever $B=$ $\mathscr{U} \times \mathbb{C}$, either $\mathfrak{B} \cong \mathscr{U}$ or $\mathbb{B} \cong \mathbb{C}$. A set $A \subseteq|\mathfrak{B}|$ disjointly generates $B$ iff each $b \in|\mathscr{B}|$ is the sum of a pairwise disjoint finite subset of $A . B$ is primitive iff $B$ is pseudo-indecomposable and is disjointly generated by the set of all $b \in \mathscr{B}$ such that $\mathscr{B}[b]$ is pseudo-indecomposable.

William Hanf has introduced a notion of "structuring" for primitive Boolean algebras in Definition 4.1 and Lemma 4.3 of [1] which we include here with minor changes for the convenience of the present discussion:

Received by the editors March 1, 1973 and, in revised form, November 12, 1973. AMS (MOS) subject classifications (1970). Primary 06A40; Secondary 02J05, 06A10.

1 The work for this paper was inspired by a seminar conducted by William Hanf at the University of California at Berkeley, in the Fall of 1969. At the time, the author was supported by a fellowship from the National Science Foundation. 
Definition. Suppose $S$ is a countable binary relation, $B$ is a Boolean algebra, and $F$ is a relation whose domain is $|S|$, then $S$ structures $B$ with $F$ iff

(1) The range of $F$ disjointly generates $\mathscr{B}$ and contains 1 but not 0 .

(2) If $a \leq b, s F a$, and $t F b$, then $s S t$ or $s=t$.

(3) If $a \dot{+} b \leq c$ and $a, b, c \in F^{\curlyvee}[s]$, then $s S s$.

(4') If $a+b=c$, then $F[a] \cup F[b] \supseteq F[c]$.

$\left(5^{\prime}\right)$ If $s S t$ and $t F_{c}$, then for some $a, b \in|B|, s F a, t F b$, and $a \dot{+} b=c$.

Definition 1. Like Hanf, we will call a countable transitive relation with a largest element a diagram. A diagram homomorphism $f: S \rightarrow T$ is a function such that $x S y$ implies $f(x) T f(y)$; $f$ is a right-strong homomorphism iff whenever $x T f(t)$, there is an such that $s \quad t$ and $f(s)=x$. The class of onto right-strong homomorphisms is closed under composition, so we may let RSE be the category of diagrams and onto right-strong homomorphisms.

I am indebted to the referee for pointing out the interesting work of R. S. Pierce [2]. His category 2, as given in Definitions 8.3 and 8.7, is similar to RSE. In particular, the category of finite diagrams and rightstrong homomorphisms is essentially the category of all finite Q.O. systems which have smallest elements. For each finite diagram $S$, let $<_{S}=$ $\{\langle y, x\rangle \mid x S y$ or $x=y\}$, and let $P_{S}=\{x \in|S| \mid x S x\}$. Then the map $S \mapsto$ $\left\langle|S|,<_{S}, P_{S}\right\rangle$ induces the indicated isomorphism, as is easily checked.

Lemma 2. Suppose $g: S \rightarrow T$ is an RSE map and $S$ structures $B$ with $F$, then $T$ structures $B$ with $g\urcorner F$.

Proof. We need to show $g^{\sim F}$ satisfies conditions (1)- $\left(5^{\prime}\right)$. Conditions (1), (2), and (4') are straightforward. For (3), suppose that $a, b, c \in 4^{\sim} g[s]$ and $a \dot{+} b \leq c$. Pick $u, v, w \in|S|$ so that $s g \smile u F, s g \smile v F b$, and $s g^{\smile} w$ $F c$. By (2) and the fact that $a, b \leq c$, we have $u S w$ or $u=w$, and $v S w$ or $v=w$. If $u S w$ or $v S w$, then $s T$ s since $s=g(u)=g(v)=g(w)$. On the other hand, if $u=v=w$, then $a, b, c \in F^{\sim}[u]$ and by (3), $u S u$, and thus $s T s$. For $\left(5^{\prime}\right)$, suppose that $s T t$ and $t g^{\vee} F c$; choose $v \in|S|$ so that $t g^{\sim} v F c$. Since $g$ is right-strong, we can also choose $u \in|S|$ so that $g(u)=s$ and $u S v$. Then for some $a, b \in|B|, u F a, v F b$, and $a \dot{+} b=c$, by $\left(S^{\prime}\right)$ applied to $S$; and, of course, $s g^{\smile} F a$ and $t g^{\smile} F b$.

Although the converse of the above lemma is false, the following lem$\mathrm{ma}$ is in the same spirit, and leads to an alternate proof of the fact that every diagram structures a Boolean algebra (see Hanf's Theorem 7.2). 
Definition 3. If $S$ is a diagram, let $S^{+}=S \cup\{\langle x, x\rangle|x \in| S \mid\}$, and let $\mathscr{U}(S)$, the ideal algebra on $S$, be the Boolean algebra of sets generated by $\left\{S^{+}[x]|x \in| S \mid\right\}$. $S$ is an irreflexive tree diagram iff $\forall x \in|S|$, not $x S x$, and $\forall x, y, z \in|S|,(x S y$ and $x S z)$ imply $\left(y S^{+} z\right.$ or $\left.z S^{+} y\right)$.

Definition 4. An $R S E$ map $g: S \rightarrow T$ has large collapse iff $\forall x \in|S|$, no finite nonempty antichain $A$ in $S[x]$ is maximal in $S[x] \cap g\left[g^{\smile}[A]\right]$.

Lemma 5. Suppose $g: S \rightarrow T$ has large collapse and $S$ is an irreflexive tree diagram, then $T$ with $g^{\smile} h^{\sim}$ structures $\mathscr{U}(S)$, where $\forall x \in|S|, \forall y_{1}, \ldots$, $y_{n} \in S[x], h\left(S^{+}[x]-\left(S^{+}\left[y_{1}\right] \cup \ldots \cup S^{+}\left[y_{n}\right]\right)\right)=x$.

Proof. It is easy to verify that $S$ with $h^{2}$ satisfies conditions (1)-( $\left.4^{\prime}\right)$; thus so does $T$ with $g^{`} h^{\swarrow}$, as in the previous lemma. For ( $\left.5^{\prime}\right)$, suppose $s T t$ and $t g^{\sim} h^{\smile} c$; then for some $y_{1}, \cdots, y_{n} \in S[h(c)]$,

$$
c=S^{+}[h(c)]-\left(S^{+}\left[y_{1}\right] \cup \cdots \cup S^{+}\left[y_{n}\right]\right) .
$$

We may assume further that $\left\{y_{1}, \ldots, y_{n}\right\}$ is an antichain since $S$ is tree ordered. We wish to choose $u$ so that $u S h(c), g(u)=s$, and $u$ is not $S$ related to any $y_{i}$ :

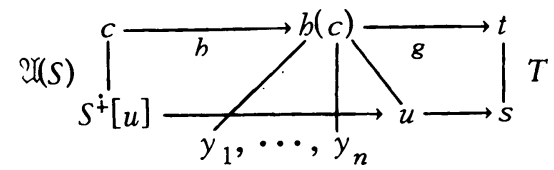

Let $A$ be the set of $y_{i}$ 's for which $s T^{+} g\left(y_{i}\right)$. If $A$ is empty, we can choose $u$ so that $u S h(c), g(u)=s$, and no $y_{i}$ is below $u$, since $S$ is a tree diagram and $g[s] \cap S[h(c)]$ is infinite by the strong collapse property. Such a point $u$ will not lie below any $y_{i}$ either, since otherwise $u S y_{i}$ implies $s T^{+} g\left(y_{i}\right)$. If $A$ is not empty, it can be extended to an infinite antichain $A^{\prime} \subseteq S[h(c)] \cap g\left[g^{-}[A]\right]$, by strong collapse. Since $S$ is a tree we can choose $v \in A^{\prime}-A$ so that no $y_{i}$ belongs to $S[v] . v$ is not below any $v_{i}$ either, for suppose $v S^{+} y_{i}$ : because $v \in A^{\prime}$, there is some $y_{j} \in A$ such that $g(v)=g\left(y_{j}\right)$, so that $g\left(y_{j}\right) T^{+} g\left(y_{i}\right)$. Since $y_{j} \in A, s T^{+} g\left(y_{j}\right)$, and thus $s T^{+} g\left(y_{i}\right)$, so that $y_{i} \in A$; but $v$ is not $S$-related to any element of $A$, a contradiction. Either $s=g(v)$ or $s T g(v)$ by choice of $v$. If $s T g(v)$, pick $u$ so that $g(u)=s$ and $u S v$; if $s=g(v)$, let $u=v$. Then since $S$ is a tree, $u$ is not $S$-related to any $y_{i}$ either, and $u S^{+} v S h(c)$. From this it is clear that $c=\left(c-S^{+}[u]\right)+S^{+}[u]$, and of course $g\left(h\left(S^{+}[u]\right)\right)=g(u)=s$, and $g\left(h\left(c-S^{+}[u]\right)\right)=g(h(c))=t$.

Construction 6. Every diagram $S$ is the range of an RSE map $\mu$ : $M T(S) \rightarrow S$ with large collapse, where $M T(S)$ is an irreflexive tree diagram, and $\mu$ and $M T(S)$ are recursive in $S$. 
Proof. Let $S$ be a diagram. Let $R$ be the irreflexive diagram given by $|R|=\{\langle x, j\rangle \mid x S x, j \in \omega\} \cup\{\langle x, 0\rangle \mid$ not $x S x\}$, and $\langle x, j\rangle R\langle y, k\rangle$ iff ( $x S y$ and not $y S x$ ) or ( $x S y$ and $y S x$ and $k<j$ ). Let $p_{1}: R \rightarrow S$ be the first coordinate projection. Then $R$ is irreflexive, and $p_{1}$ is rightstrong. Let $|Q|=\left\{\left\langle 1_{R}, 0\right\rangle\right\} \cup\left(|R|-\left\{1_{R}\right\}\right) \times \omega$ and let $\langle x, j\rangle Q\langle y, k\rangle$ iff $x Q y$; let $p_{2}: Q \rightarrow R$ be the first coordinate projection. Then $p_{2}$ is right-strong and has large collapse. Finally, let $|M T(S)|$ be the set of all finite $Q$-chains with largest element 1 ; demand of $M T(S)$ only that whenever $\left\langle x_{1}, \ldots, x_{j}, \ldots, 1\right\rangle$ is a $Q$-chain, we have $\left\langle x_{1}, \ldots, 1\right\rangle M T(S)$ $\left\langle x_{j}, \ldots, 1\right\rangle$. Let $p_{3}: M T(S) \rightarrow Q$ be given by $p_{3}\left(x_{1}, \ldots, 1\right)=x_{1}$. Then $M T(S)$ has the prescribed properties, and $p_{3}$ is right-strong. Let $\mu=$ $p_{1} p_{2} p_{3}$; the large collapse condition carries over from $p_{2}$ to $\mu$. Finally, it is clear from the construction that $\mu$ and $M T(S)$ are recursive in $S$.

Corollary 7. If $S$ structures a Boolean algebra $\mathscr{P}$, then $S$ structures $\mathfrak{B}$ with $f^{\sim}$ for some function $f$.

Proof. $S$ structures $\mathscr{U}(M T(S))$ with $\mu^{\smile} h^{\smile}$, where $h$ and $\mu$ are as above; by Hanf's Theorem $4.5, \mathfrak{R} \cong 2 \mathfrak{L}(M T(S))$.

Corollary 8. Every diagram $S$ structures a Boolean algebra recursively definable in $S$.

Proof. It suffices to construct an algebra isomorphic with $\mathscr{U}(M T(S))$ that is recursive in $M T(S)$. Let $\mathbf{B}$ be the set of all pairs $\langle a, A\rangle$, where $A$ is a finite antichain in $M T(S)[a]$. Then $\forall c \in b[M T(S)], \exists !\langle a, A\rangle \in \mathbf{B}$ : $c=M T(S)^{+}[a]-\bigcup\left\{M T(S)^{+}[x] \mid x \in A\right\}$. Let $|\mathscr{E}|$ be the set of all finite subsets $\left\{\left\langle a_{1}, A_{1}\right\rangle, \ldots,\left\langle a_{n}, A_{n}\right\rangle\right\}$ of $B$ such that if for some $i, j, a_{i} M T(S)^{+}$ $a_{j}$; then for some $b \in A_{i}, a_{i} M T(S) b$. Each element of $\mathscr{L}(M T(S))$ is uniquely represented as the disjoint sum of elements of $b[M T(S)]$, and thus is uniquely represented by an element of $|\mathscr{P}|$. The recursiveness in $M T(S)$ of the Boolean operations on $\mathscr{E}$ is now straightforward. To compute the join operation, for example, first define the meet of two finite antichains $A, B$, by $A \wedge B=\{x \in A \cup B \mid x \in A \cap B$ or $\exists y \in A \cup B, x M T(S) y\}$. Then to find the join of a finite subset $\left\{\left\langle a_{1}, A_{1}\right\rangle, \ldots,\left\langle a_{n}, A_{n}\right\rangle\right\}$ of $B$, just replace each pair $\left\langle a_{i}, A_{i}\right\rangle$ and $\left\langle a_{j}, A_{j}\right\rangle$ with $\left\langle a_{j}, A_{i} \wedge A_{j}\right\rangle$ whenever $a_{i} M T(S)^{+} a_{j}$ and $\forall b \in A_{j}$, not $a_{i} M T(S) b$. Finally, the join of two elements from $\mathscr{P}$ is the join of their union.

2. Minimal and maximal diagrams. Consider $R S E$ to be preordered by the relation $Q \leq R$ iff there is an $R S E$ map from $R$ to $Q$. The $R S E$ 
minimal diagrams turn out to be just those of the type $S(\mathbb{R})$ given in Hanf's Construction 5.3, which for convenience, we restate as follows: Suppose $\mathscr{Z}$ is a primitive Boolean algebra. Let $P I(\mathscr{P})=\{b \in|\mathscr{P}| \mid \mathscr{E}[b]$ is pseudo-indecomposable\}. $\forall a \in P I(\mathscr{P})$, let $\pi(a)=\{b \mid \mathscr{B}[b] \cong \mathscr{P}[a]\}$. Let $|S(\mathscr{B})|=\{r(a) \mid a \epsilon$ $P I(\mathscr{E})\}$, and $\forall a, b \in P I(\mathbb{E})$, let $r(a) S(\mathbb{B}) r(b)$ iff $\mathbb{E}[a] \times \mathbb{R}[b] \cong \mathbb{R}[b]$. The following result is analogous to Pierce's Lemma 9.1.

Theorem 9. If a diagram $R$ structures a Boolean algebra $\mathbb{B}$, then there is a unique RSE map $\pi$ from $R$ to $S(\mathbb{B})$.

Proof. Assume $R$ structures $\mathscr{P}$ with $F$. We shall show that $\pi=F r$ is the required $R S E$ map from $R$ to $S(\mathscr{P})$.

Step I. $\pi=F_{\tau}$ is well defined: Suppose $s F a$ and $s F b$; then by Hanf's Lemma $4.4, \mathbb{B}[a] \cong \mathscr{P}[b]$. By the proof of Hanf's Theorem 5.2, $a, b \in P I(B)$, and thus $r(a)=r(b)$.

Step II. $\pi$ is onto; that is, if $b \in P I(B)$, then $\exists b^{\prime} \in P I(\mathscr{B})$, js $\in|R|$, $s F b^{\prime}$ and $x(b)=\pi\left(b^{\prime}\right)$ : By condition (1) of the structure definition, $b=$ $b_{1}+\cdots+b_{n}$ with $b_{1}, \ldots, b_{n}$ in the range of $F$, and for some $b_{i}, \mathscr{E}[b] \cong$ $\mathfrak{Q}\left[b_{i}\right]$ since $\mathbb{E}[b]$ is pseudo-indecomposable.

Step III. $\pi$ is a homomorphism; that is, if $s R t$, then for some $a, b \in$ $|\mathbb{P}|, s F a, t F b$, and $\mathbb{E}[a] \times \mathbb{E}[b] \cong \mathbb{B}[b]$. Pick $c$ so that $t F c$. Then by condition $\left(5^{\prime}\right)$ of the structure definition, we can choose $a, b$ so that $s F a, t F b$, and $a+b=c$; then by Hanf's Lemma 4.4,

$$
\mathfrak{B}[b] \cong \mathfrak{B}[c]=\mathfrak{B}[a+b] \cong \mathfrak{B}[a] \times \mathfrak{B}[b] .
$$

Step IV. $\pi$ is right-strong: Suppose $\pi(s) S(\mathbb{P}) \pi(t)$. Then we may choose $a, b$ so that $s F a$ and $t F b$; in which case $\pi(a)=\pi(s) S(\mathbb{B}) \pi(t)=\pi(b)$, so that $\mathscr{E}[a] \times \mathfrak{B}[b] \cong \mathscr{E}[b]$. Hence we may write $b=a_{1}+b_{1}$, where $r\left(a_{1}\right)=$ $\pi(a)$ and $r\left(b_{1}\right)=r(b)$. By condition (1) of the structure definition we may write $a_{1}=a_{2}+c$ and $b_{1}=b_{2} \dot{+} d$, where $r\left(a_{1}\right)=r\left(a_{2}\right), r\left(b_{1}\right)=r\left(b_{2}\right)$, and $a_{2}, b_{2}$ are in the range of $F$. Choose $s^{\prime}, t^{\prime}$ so that $s^{\prime} F a_{2}$ and $t^{\prime} F b_{2}$; then $\pi\left(s^{\prime}\right)=r(a)=\pi(s)$ and $\pi\left(t^{\prime}\right)=r(b)=\pi(t)$. Since $b \geq a_{2}+b_{2}$, we have ( $s^{\prime} R t$ or $\left.s^{\prime}=t\right)$ and $\left(t^{\prime} R t\right.$ or $\left.t^{\prime}=t\right)$ by condition (2) of the structure definition. If $s^{\prime} R t$, we are done; suppose $s^{\prime}=t$. If $t^{\prime}=t$, then since $b \geq a_{2}+b_{2}$, we have $s^{\prime}=t R t$ by condition (3). If $t^{\prime} R t$, however, set $b_{2}=b_{3}+a_{3}$, with $r\left(b_{3}\right)=r\left(b_{2}\right)=r(b)$ and $r\left(a_{3}\right)=r(a)$; as before, set $a_{3}=$ $a_{4}+a_{5}$, with $\lambda\left(a_{3}\right)=\pi\left(a_{4}\right)$, and $a_{4}$ in the range of $F$. Pick $s^{\prime \prime}$ so that $s^{\prime \prime} F a_{4}$. Then by condition (2), $s^{\prime \prime} R t^{\prime}$ or $s^{\prime \prime}=t^{\prime}$, since $a_{4} \leq b_{2}$. In either case $s^{\prime \prime} R t$, and $\pi\left(s^{\prime \prime}\right)=\pi\left(a_{4}\right)=\pi(a)=\pi(s)$. 
Step V. $\pi$ is unique: First, $\pi=F \tau$ is independent of $F$; that is, if $R$ structures $B$ with $F$ and with $G$, then $F r=G r$ : Suppose $s F a$ and $s G b$; then by Hanf's Lemma 4.4, $B[a] \cong \mathscr{E}[b]$, and thus $\gamma(a)=\pi(b)$. Next, if $S(\mathscr{E})$ structures $\mathscr{Q}$ with $H$, then $H \subseteq \tau^{2}: S(\mathscr{Q})$ structures $\mathscr{B}$ with $\tau^{2}$, as is shown in the proof of Hanf's Theorem 5.4. Also, $H \tau$ is independent of $H$, by what we have just seen (in the special case where $R=S(B)$ ). Hence $H \tau=\tau^{\sim} \tau$. But $\tau^{\sim} \tau$ is the identity map on $S(\mathscr{P})$, and thus $H \subseteq \tau^{`}$. Finally, if $g$ is an

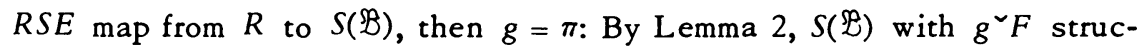
tures $B$. Hence $\left.g^{\vee} F \subseteq \tau\right\urcorner$, in which case $F^{\vee} g \subseteq \tau$, and thus $g \subseteq F F^{\vee} g \subseteq$ $F \tau=\pi$. But $g$ and $\pi$ are functions with the same domain; so $g=\pi$.

Corollary 10. The RSE minimal diagrams are just those of the type given in Hanf's Construction 5.3. They are essentially the irreducible simple P.O. systems of Pierce.

Proof. For the first statement, suppose that $f: S(\mathbb{Z}) \rightarrow R$ is an $R S E$ map. Then $R$ structures $\mathscr{P}$ by Lemma 2 and Hanf's Theorem 5.4. Hence, we need only let $\pi: R \rightarrow S(\mathscr{B})$ be the unique map given above. Notice that $f \pi$, being the unique $R S E$ map from $S(\mathbb{B})$ to $S(\mathbb{B})$, is the identity; consequently, since $f$ is onto, it is an isomorphism. The second statement now follows from the definitions supplied with Pierce's Propositions 8.13 and 8.14 , and the fact that every right-strong homomorphism factors through an $R S E$ map.

Corollary 11. The relation "S structures $\mathbb{D}$ " induces a 1-1 correspondence between the components of RSE and the isomorphism types of primitive Boolean algebras.

Proof. If $S$ and $T$ structure the Boolean algebra $B$, then by the above theorem, both precede $S(\mathbb{R})$ in $R S E$. Conversely, suppose $f: Q \rightarrow R$ is an $R S E$ map. If $Q$ structures $B$, then so does $R$ by Lemma 2 . On the other hand, if $R$ structures $\mathscr{B}$, then $Q$ structures $\mathscr{U}(M T(Q))$ by Construction 6 , $R$ structures $\mathscr{U}(M T(Q))$ by Lemma 2 , and $\mathscr{U}(M T(Q)) \cong \mathscr{B}$ by Hanf's Lemma 4.4; hence $Q$ also structures $B$. A finite repetition of this argument shows that if $S$ and $T$ lie in the same component of $R S E$, they structure the same Boolean algebras.

Lemma 12. Suppose $R$ structures $\mathbb{B}, \pi: R \rightarrow S(\mathscr{B})$ is the unique $R S E$ map, $x, y \in|R|$, and $R[x] \cong R[y]$ (or more accurately, $R|R[x] \cong R| R[y]$ ). Then $\pi(x)=\pi(y)$. 
Proof. $\forall x, y \in|R|$, set $x \sim y$ iff $R[x] \cong R[y]$. Then the projection map from $R$ to $R / \sim$ is easily seen to be right-strong. Consequently, it must factor through $\pi$.

Theorem 13. For any primitive Boolean algebra $\mathbb{B}$, there is (up to isomorphism) a unique tree diagram $P$ that structures $\mathbb{B}$ and satisfies the following equivalent conditions:

(i) $P$ is RSE maximal.

(ii) $P$ is of the type given in Construction 6.

(iii) $P$ is irreflexive, and $\forall x \in|P|, P^{\smile}[x]$ is finite and $\forall y \in P[x]$, there exist infinitely many $z \in P[x]$ such that $P[z]$ is a maximal ideal in $P[x]$ isomorphic with $P[y]$.

Proof. The diagrams discussed will structure $\mathscr{R}$, unless otherwise indicated. The first step is to show that if $P$ is a tree diagram, $S$ satisfies (iii), and $f: P \rightarrow S$ is an $R S E$ map, then $P \cong S:$ First, $P$ must be irreflexive since $S$ is. Also, $\forall x, f \mid P^{`}[x]$ must be an embedding, and thus $P^{\smile}[x]$ is finite. For each $s \in|S|$, let $B(s)$ be the set of all $s^{\prime} \in|S|$ such that $S\left[s^{\prime}\right] \cong S[s]$, and $s$ and $s^{\prime}$ have the same $S$-successor. For each $x \in|P|$, let $A(x)$ be the set of all $x^{\prime} \in|P|$ such that $S[f(x)] \cong S\left[f\left(x^{\prime}\right)\right]$ and $x$ and $x^{\prime}$ have the same $P$-successor. We can define an isomorphism $g: P \rightarrow S$ inductively as follows: let $g(1)=1$. Assume that for $n>0, g$ has been defined on $D_{n}=\left\{y \mid\left(P^{\smile}[y]\right)^{=}=n\right\}$, and that for each $y \in D_{n}, S[g(y)] \cong S[f(y)]$. Extend $g$ to each $x$ such that $\left(P^{\complement}[x]\right)^{=}=n$ as follows: Suppose $y$ is the $P$-successor of $x$; then $f(x) S f(y)$. By (iii) we may choose $s_{0}$ so that $S\left[s_{0}\right] \cong S[f(x)]$ and $f(y)$ is the $S$-successor of $s_{0}$. By assumption, $S[f(y)]=$ $S[g(y)]$, and thus we may choose $s$ so that $S[s] \cong S\left[s_{0}\right]$ and $g(y)$ is the $S$-successor of $s$. $B(s)$ is infinite by (iii). So is $B\left(s_{0}\right)$; for each $s^{\prime} \epsilon$ $B\left(s_{0}\right)$, there is an $x^{\prime} \in P$ such that $f\left(x^{\prime}\right)=s^{\prime}$ and $x^{\prime} P y$, since $s^{\prime} s f(y)$ and $f$ is right-strong; furthermore, $y$ is the $P$-successor of $x^{\prime}$ since $f(y)$ is the $S$-successor of $s^{\prime}$. Each such $x^{\prime}$ belongs to $A(x)$; hence $A(x)$ is infinite. Extend $g$ to $A(x)$ in any way such that $g \mid A(x)$ is a 1-1 correspondence onto $B(s)$. In this way $g$ may be extended to the set of all $x$ such that $\left(S^{\sim}[x]\right)^{=}=n$ since both of the families $\{A(x)|x \in| P \mid\}$ and $\{B(s)|s \in| S \mid\}$ are pairwise disjoint. The completed map $q: P \rightarrow S$ so obtained will be an isomorphism by construction.

A moment's thought shows that the diagrams given in Construction 6 satisfy (iii); thus (ii) $\rightarrow$ (iii). The above paragraph shows directly that (iii) $\rightarrow$ (i). Suppose $P$ is maximal; then in $R S E, P$ precedes $M T(P)$ since $M T(P)$ precedes $P$, and thus $P \cong M T(P)$ since $M T(P)$ satisfies (iii); 
hence $(\mathrm{i}) \rightarrow$ (ii). Finally, uniqueness may be shown as follows: Suppose $Q, R$ satisfy (iii), let $S=S(\mathbb{B})$, and let $\pi: Q \rightarrow S$ and $\rho: R \rightarrow S$ be the unique $R S E$ maps. We may define an isomorphism $\phi: Q \rightarrow R$ by a back and forth argument as follows: Since $\forall x \in|Q|, Q^{\smile[x]}$ is finite, we may let $\left\{x_{i} \mid i \in \omega\right\}$ be an enumeration of $|Q|$ such that $\forall x_{i}, Q^{\smile}\left[x_{i}\right] \subseteq\left\{x_{j} \mid j<i\right\}$. Let $\left\{y_{i} \mid i \in \omega\right\}$ be a similar enumeration of $|R|$. Let $\phi_{0}\left(1_{Q}\right)=1_{R}$. Assume a partial isomorphism $\phi_{j}$ from a finite subset of $|Q|$ to $|R|$ has been defined, that for

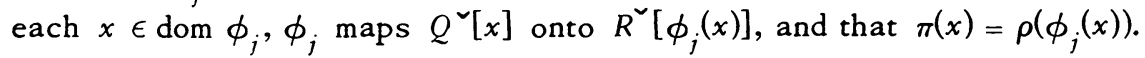
To extend $\phi_{j}$ to the next element, say $x_{i} \in|Q|$ for example, let $x$ be the $Q$-successor of $x_{i \cdot}$. Then $\pi\left(x_{i}\right) S \pi(x)=\rho\left(\phi_{j}(x)\right)$, and we may choose $y \epsilon$ $|R|$ so that $y R \phi_{j}(x)$ and $\rho(y)=\pi\left(x_{i}\right)$. Then there are infinitely many points $z \in R\left[\phi_{j}(x)\right]$ such that $\phi_{j}(x)$ is the $R$-successor of $z$ and $R[y] \cong$ $R[z]$; let $\phi_{j+1}\left(x_{i}\right)$ be one such $z$ that does not belong to the range of $\phi_{j} \cdot$ Since $R\left[\phi_{j+1}\left(x_{i}\right)\right] \cong R[y], \rho\left(\phi_{j+1}\left(x_{i}\right)\right)=\rho(y)=\pi\left(x_{i}\right)$, by the above lemma; $\phi_{j+1}$ maps $Q^{\sim}\left[x_{i}\right]$ onto $R^{\smile}\left[\phi_{j+1}\left(x_{i}\right)\right]$, and it is clearly a partial isomorphism. $\phi$, the union of the $\phi_{j}^{\prime}$ 's is the desired isomorphism.

Pierce's Proposition 10.2 suggests the following analogue for diagrams and primitive Boolean algebras: Define the product of two diagrams $S, T$ by $(s, t) S \times T\left(s^{\prime}, t^{\prime}\right)$ iff $\left(s S^{+} s^{\prime}\right.$ and $\left.t T^{+} t^{\prime}\right)$ and $\left(s S s^{\prime}\right.$ or $\left.t T t^{\prime}\right)$. For Boolean algebras $\mathfrak{U}, \mathfrak{B}$, let $\mathfrak{U} \oplus \mathfrak{B}$ denote the coproduct of $\mathfrak{U}$ and $\mathfrak{B}$ in the category of Boolean algebras and homomorphisms that preserve 1 .

Proposition 14. If $S$ structures $\mathscr{Q}$ and $T$ structures $\mathscr{B}$, then $S \times T$ structures $\mathscr{U} \oplus \mathbb{P}$.

Proof. Suppose $S$ structures $\mathscr{U}$ with $F$ and $T$ structures $\mathscr{B}$ with $G$. We may consider $\mathscr{U} \oplus \Re$ to consist of all formal sums $a_{1} b_{1}+\cdots+a_{n} b_{n}$ where $\forall i<j$, either $a_{i} a_{j}=0$ or $b_{i} b_{j}=0$, modulo the usual identities for Boolean algebras. It is now a straightforward task to show that $S \times T$, $F \times G$, and $\mathscr{U} \oplus \mathscr{P}$ satisfy the structure definition, except perhaps for condition $\left(5^{\prime}\right)$ : Assume that $(s, t) S \times T\left(s^{\prime}, t^{\prime}\right)$ and $\left(s^{\prime}, t^{\prime}\right) F \times G a^{\prime \prime} b^{\prime \prime}$. Then $s^{\prime} S a^{\prime \prime}, t^{\prime} G b^{\prime \prime}$, and ( $s S s^{\prime}$ and $t T^{+} t^{\prime}$ ) or vice versa. If $s S s^{\prime}$ and $t=$ $t^{\prime}$, then there are $a, a^{\prime} \in|\mathcal{Q l}|$ such that $s F a, s^{\prime} F a^{\prime}$, and $a+a^{\prime}=a^{\prime \prime}$. But then $(s, t) F \times G\left(a, b^{\prime \prime}\right),\left(s^{\prime}, t^{\prime}\right) F \times G\left(a^{\prime}, b^{\prime \prime}\right)$, and $a b^{\prime \prime}+a^{\prime} b^{\prime \prime}=a^{\prime \prime} b^{\prime \prime}$. In the other interesting case, if $s S s^{\prime}$ and $t T t^{\prime}$, then there are $a, a^{\prime} \epsilon$ $|\mathfrak{X}|$ and $b, b^{\prime} \in|\mathbb{P}|$ such that $s F a, s^{\prime} F a^{\prime}, t G b, t^{\prime} G b^{\prime}, a+a^{\prime}=a^{\prime \prime}$, and $b+b^{\prime}=b^{\prime \prime}$, so that

$$
a b+a^{\prime} b^{\prime} \leq a b+a^{\prime} \cdot b \dot{+} a b^{\prime}+a^{\prime} \cdot b^{\prime}=a^{\prime \prime} b^{\prime \prime},
$$


and $(s, t) F \times G a b$ and $\left(s^{\prime}, t^{\prime}\right) F \times G a^{\prime} b^{\prime}$; by Hanf's Lemma 4.3, this is good enough.

The above result is interesting because it suggests that diagrams structure Boolean algebras contravariantly (as does the pairing of the embedding $S[s] \subseteq S[1]$ with the projection $\mathscr{U} \rightarrow \mathscr{U}[a]$, where $S$ structures $\mathscr{U}$ and $S \mid S[s]$ structures $\mathscr{U}[a])$. As with Pierce's products of P.O. systems, the above diagram product has not yet been placed in a correct categorical framework. Perhaps there is a product-preserving contravariant functor from a category containing $R S E$ to a suitable category of isomorphism types of Boolean algebras.

\section{REFERENCES}

1. William Hanf, Primitive Boolean algebras, Proc. Sympos. Pure Math., vol. 25, Amer. Math. Soc., Providence, R. I., 1974, pp. 75-90.

2. R. S. Pierce, Compact zero-dimensional metric spaces of finite type, Mem. Amer. Math. Soc. No. 130 (1970).

DEPARTMENT OF MATHEMATICS, BOWLING GREEN STATE UNIVERSITY, BOWLING GREEN, OHIO 43403 